Cahiers $d u$ MONDE RUSSE

\section{Cahiers du monde russe}

Russie - Empire russe - Union soviétique et États indépendants

45/3-4 | 2004

Varia

\title{
Arlen V. Bljum, Vladimir G. Volovnikov, eds., Cenzura v Sovetskom sojuze, 1917-1991
}

\section{Benjamin Guichard}

\section{OpenEdition}

\section{Journals}

Édition électronique

URL : https://journals.openedition.org/monderusse/4212

DOI : $10.4000 /$ monderusse.4212

ISSN : $1777-5388$

Éditeur

Éditions de l'EHESS

\section{Édition imprimée}

Date de publication : 1 juillet 2004

Pagination : 725-728

ISBN : 2-7132-2009-2

ISSN : $1252-6576$

Référence électronique

Benjamin Guichard, «Arlen V. Bljum, Vladimir G. Volovnikov, eds., Cenzura v Sovetskom sojuze, 1917-1991 », Cahiers du monde russe [En ligne], 45/3-4 | 2004, mis en ligne le 03 juin 2009, consulté le 02 septembre 2022. URL : http://journals.openedition.org/monderusse/4212 ; DOI : https://doi.org/ $10.4000 /$ monderusse. 4212

Ce document a été généré automatiquement le 2 septembre 2022

Tous droits réservés 


\title{
Arlen V. Bljum, Vladimir G. Volovnikov, eds., Cenzura v Sovetskom sojuze, 1917-1991
}

\author{
Benjamin Guichard
}

\section{RÉFÉRENCE}

Arlen V. BLJUM, Vladimir G. VOLOVNIKOV, eds., Cenzura v Sovetskom sojuze,

1917-1991. Dokumenty (La censure en Union soviétique 1917-1991. Documents).

Moscou, ROSSPEN, 2004, 576 p. (Kul'tura i vlast' ot Stalina do Gorbačeva. Dokumenty).

1 Fruit d'une collaboration entre la Direction des archives de la Fédération de Russie et l'Institut Lotman de l'université de Bochum, la collection de l'éditeur Rosspen consacrée aux rapports entre la culture et le pouvoir de Stalin à Gorbačev propose, deux ans après la synthèse de Tamara M. Gorjaeva ${ }^{1}$, un recueil de documents consacré à la censure tout au long de la période soviétique. Les deux ouvrages ne se répondent cependant pas. Les documents publiés par Arlen Bljum ont en effet été réunis par l'auteur dans le cadre de ses propres recherches sur l'histoire de la censure qui, à ce jour, ont donné lieu à la publication de deux premiers volumes².

2 Le volume proposé ici par Arlen Bljum a donc été composé au début des années 1990 et les documents rassemblés furent publiés une première fois, de façon assez confidentielle, en Allemagne, il y a cinq ans ${ }^{3}$. Ce décalage explique deux limites du recueil. Le fonds du Glavlit, organisme central en charge de la supervision de la censure de 1922 à 1991, n'est pas représenté car il était alors inaccessible. Par ailleurs, alors que les chantiers ouverts ces dix dernières années ont éclairé l'action de la censure dans le domaine du théâtre, du cinéma ou de la radio, la documentation rassemblée ici privilégie nettement le contrôle des imprimés : sur un ensemble de 459 documents, seuls 13 n'ont pas trait à la littérature et à la presse. 
3 Le recueil propose un appareil critique soigné ${ }^{4}$, assorti d'un double index facilitant la consultation et enrichi de notes rédigées par V.G. Volovnikov. Ce chercheur de l'université de Bochum a notamment actualisé la sélection de documents en citant de nombreux matériaux mis au jour ces dernières années. L'ouvrage offre des indications factuelles et biographiques, utiles notamment pour préciser le sort des publications incriminées. On peut toutefois regretter que ces notes développent parfois une lecture simpliste : ainsi telle protestation de Trockij contre les excès de la censure militaire en janvier 1919 (doc. 8) est commentée par trois lignes de notice biographique et quatorze d'accusations contre le personnage, lui attribuant la paternité de l'expression « camp de concentration » et soulignant sa responsabilité dans la politique de déportation. En guise de renseignements sur la position politique de Trockij à la date du document, d'éléments susceptibles d'éclairer le rôle d'une telle dénonciation dans une éventuelle lutte d'influence ou volonté de défendre l'emprise de son commissariat sur une fonction de contrôle disputée par le Narkompros, le commentateur nous offre donc une charge manichéenne destinée à rappeler, si l'on en doutait, que Trockij n'était pas un libéral épris de liberté d'expression mais un méchant totalitaire.

4 Ces limites posées, il faut souligner le nombre et la qualité des documents présentés dans un ordre strictement chronologique et qui parviennent à bien illustrer les caractéristiques de la censure soviétique. Arlen Bljum est parvenu à contourner les principales difficultés d'accès aux archives centrales de façon originale. De nombreux recueils ont en effet choisi de privilégier les archives du parti, notamment celles du Département de la propagande, dans un souci de remonter à la source des décisions ${ }^{5}$. Arlen Bljum a préféré se concentrer sur le fonctionnement de l'administration en charge de la censure en privilégiant les archives de la branche de Leningrad du Glavlit, le Lengublit. Celles-ci permettent à la fois une analyse locale et la reconstitution d'un corpus d'instructions émises par le centre car la correspondance avec Moscou a été soigneusement conservée. Plus de la moitié des documents reproduits est issue de ce fonds. Les premières années du Glavlit sont également éclairées par les archives personnelles de son premier responsable, P. I. Lebedev-Poljanskij, conservées à l'Académie des sciences. Les fonds du GARF, des archives du parti de Leningrad ou du RGASPI ont également été utilisés, ces derniers notamment au sujet du rapatriement des archives et des bibliothèques trophées après la guerre (doc. 270-278). Seulement 15 documents avaient fait l'objet d'une publication antérieure. L'ouvrage présente donc une documentation inédite qui permet de croiser les décisions du centre et leur mise en œuvre à l'échelle locale de 1917 à 1991.

5 Si la majeure partie du recueil suit donc pas à pas l'activité du Glavlit, les premiers documents s'efforcent de démontrer la diversité des formes de censure mises en place de façon précoce par le jeune État soviétique, au sein des différents soviets locaux, de l'armée et du Narkompros. Arlen Bljum reproduit notamment plusieurs notes de lecture internes du Gosizdat qu'il assimile donc à des rapports de censure (doc. 11, 12, 14 et 18): cette source lui avait permis notamment de souligner l'implication de Valerij Brjusov dans la mise en place des organismes de contrôle, une interprétation des engagements politiques du poète symboliste qui lui avait valu de sévères critiques lors de la publication de son premier livre ${ }^{6}$. S'il faut bien sûr noter le risque d'assimiler toute forme de choix et de critique éditoriale à un acte de censure et de contrôle administratif, la nature très politique des arguments employés dans ces notes privilégie l'interprétation de l'auteur. Il faut notamment ajouter que, dans un contexte de pénurie, 
le Gosizdat avait un pouvoir presque total dans l'attribution des stocks de papier et les réquisitions d'imprimerie aux différentes maisons d'édition: un refus de son comité éditorial pouvait donc s'apparenter à une condamnation.

6 La mise en place du Glavlit en 1922 est illustrée par une correspondance très vive entre le responsable du Narkompros A. V.Lunačarskij et P. I. Lebedev-Poljanskij. Appuyé par le département de la propagande du Comité central, ce dernier parvient à affranchir le Glavlit de la tutelle du Narkompos et à renforcer le pouvoir de son agence, notamment face à la concurrence de la censure militaire. Après le Dégel, le rôle prépondérant du KGB dans lutte contre la dissidence contribue à l'inverse à fragiliser l'indépendance du Glavlit (doc. 353, 383-393 notamment).

7 Le fonctionnement interne de la censure est également éclairé par cette documentation. Les purges de 1937-1938 bouleversent ainsi considérablement l'organisation du Lengublit (doc. 223) et visent en priorité les censeurs qui manient les langues étrangères (doc. 229). À l'inverse, la période de la guerre se traduit par une certaine valorisation des fonctions de censeurs, certains étant même médaillés pour leurs services à la fin du conflit (doc. 269). La période de la guerre est aussi celle d'une évolution dans le vocabulaire de la censure : alors que l'action de celle-ci est justifiée depuis la révolution par des motifs politiques et idéologiques, à partir des années 1940, la notion de secret d'État et d'intérêt national semble s'y substituer. La période du Dégel, en revanche, ne semble pas être l'occasion de changements considérables dans l'organisation de cette administration. Mais les rapports du début des années 1960 montrent que l'attitude de la société, elle, a changé: le Lengublit semble confronté de plus en plus souvent à des stratégies de contournement de la réglementation de la part des rédactions (doc. 330 à 338).

La fin de la période soviétique est, fait assez rare, assez bien documentée, avec près de 25 documents postérieurs à 1985. On y trouve les dernières versions de l'inventaire des informations interdites à la publication (doc. 442) et différents documents qui traduisent les tentatives d'adaptation du Glavlit aux réformes en cours. Non sans ironie, Arlen Bljum a sélectionné différents documents de l'année 1990 révélant l'effort des derniers responsables du Glavlit pour retirer des dépôts d'archives les anciens documents de travail des censeurs afin de faire disparaître les traces les plus compromettantes. Le recueil s'achève sur l'acte de dissolution du Glavlit, auquel était annexée, nous est-il précisé, la liste des documents secrets couvrant la période 1941-1991 qui ont été détruits dans les archives du Lengublit.

Ces exemples ne sont qu'une proposition de lecture de l'importante documentation rassemblée par Arlen Bljum. Celle-ci vient compléter utilement, à l'aide d'exemples locaux et de cas très concrets, des recueils de documents consacrés à la censure qui privilégiaient jusqu'ici les archives du parti ou des organismes centraux.

\section{NOTES}

1. T. M. Gorjaeva, Političeskaja cenzura v SSSR. 1917-1991 (La censure politique en URSS, 1917-1991), Moscou, ROSSPEN, 2002. 
2. A. V. Bljum, Za kulisami "Ministerstva pravdy»: tajnaja istorija sovetskoj cenzury, 1917-1929 (Derrière les coulisses du "Ministère de la vérité »: histoire secrète de la censure soviétique, 1917-1929), Saint-Pétersbourg, Akademičeskij proekt, 1994 et A. V. Bljum, Sovetskaja cenzura v epohu total'nogo terrora, 1929-1953 (La censure soviétique à l'époque de la terreur totale, 1929-1953), Saint-Pétersbourg, Akademičeskij proekt, 2000. Il faut également ajouter un article consacré au démantèlement du Glavlit : «Zakat Glavlita. Kak razrušalas' sistema sovetskoj cenzury : dokumental'naja hronika » (Le déclin du Glavlit. Comment a été démoli le système de la censure soviétique : chronique documentaire), Kniga. Issledovanija i materialy [Moscou], vol. 71, 1995, p. 168-187.

3. A. V. Bljum, Zensur in der UdSSSR. Archivdokumente. 1917-1991, Bochum, 1999, 575 p. (Dokumente und Analysen zur russischen und sowjetischen Kultur, 13/II). Cette première publication, en langue russe, accompagnait la traduction allemande du premier volume d'A. Bljum consacré à la censure soviétique.

4. Une probable coquille prive cependant de mention de sa source le document 184, p. 206.

5. Contemporain des recherches de Bljum, on peut citer D. L. Babičenko, ed., "Literaturnyj front ». Istorija političeskoj cenzury, 1932-1946 gg. Sbornik dokumentov (Le "front littéraire ». Histoire de la censure politique, 1932-1946. Recueil de documents), Moscou, Enciklopedija rossijskih dereven', 1994, 273 p. Et, plus récemment, dans la même collection que le présent recueil: Ideologičeskie komissii CK KPSS, 1958-1964: dokumenty (Les commissions idéologiques du CC du PCUS, 1958-1964: documents), Moscou, ROSSPEN, 1998; Apparat CK KPSS i kul'tura, 1953-1957 gody: dokumenty (L'appareil du CC du PCUS et la culture, 1953-1957 : documents), Moscou, ROSSPEN, 2002.

6. C'était le sens de la critique adressée par Ju. I. Glebov dans Kniga. Issledovanija i materialy, vol. $70,1995$. 